\title{
Flow field computation for simplified high voltage gas circuit breaker model by upwind meshfree method
}

\author{
Seong-Kwan Park ${ }^{1}$ \\ 1) Department of Mathematics, Yonsei University, Seoul 120-749, KOREA \\ Corresponding Author : S.-K. Park, pakskwan@yonsei.kr
}

\begin{abstract}
To effectively design high voltage (HV) circuit breakers, the upwind meshfree (UM) scheme which was proposed by Choe et al.[5] and Park et al.[6] is tested. The UM scheme is implemented to a simplified model for the HV circuit breaker and showed a robust behavior even in a severe boundary condition. A good shock capturing is shown in the computations and a delicate gas diffusion through a slit is also computed, which is usually difficult to catch. Vector splitting method is employed to exploit the merit of meshfree methods in the hyperbolic problems.
\end{abstract}

\section{INTRODUCTION}

Much progress has been made in the design of high voltage (HV) circuit breakers since 1960's although we could not cover all the detailed history and researches here. Briefly speaking, the first SF6 puffer circuit breaker appeared in 1964 and since then there have been various models to describe the physical phenomena like Swanson-Roidt[1], Hermann-Ragaller[2], Lowke-Lee[3], and Chan-Cowley-Fang[4]. However, designing a HV circuit breaker is still a complicated work in view of experiments and theories. It is a formidable task to compromise flow analysis, mathematical models to complicated phenomena, complex geometry and fast moving boundary. It is our view that now a reasonable mathematical model can be established and we can analyze the suggested model in a certain depth.

On the contrary computer simulation has become a necessary design tool to predict HV circuit breakers. It would be impossible to get optimized process without computer simulations since there are infinitely many variations. Therefore computer simulations in HV circuit breaker technology could reduce the research and development (R\&D) cost significantly and improve mathematical models theoretically. Now that numerical techniques to compute partial differential equations are well established, it is more and more important to optimize by using computer simulations.

One of the most time consuming process in implementing a numerical technique is to design a proper mesh for the computational geometry. The HV circuit breaker has a complicated shape and it is not a simple matter to find a mesh for finite element method or finite difference method. In this note we introduce a meshfree method. Meshfree method can handle large deformation of moving body and have resolution capability as well as it is robust to complex geometry.

Computer simulation of a HV circuit breaker switching involves fluid phenomena, electric field and heat diffusion process. Finding a simple model and discrete algorithm to simulate entire physics is our long term goal. As a first step, we analyze the shock phenomena and gas diffusion in a stationary HV circuit breaker using a simplified model to fully understand gas 
physics inside of a HV circuit breaker. The electric field has a much large period and thus we need to consider the fluid part and heat part in a much smaller scale. So, for our simplified model, we disregard the electric field and arc model and it is assumed that there is no energy source term due to electric field. In the forthcoming paper, we will consider the full gas dynamic model including heat source as well as moving boundary.

\section{REFERENCES}

1. B. W. SWANSON AND R. M. RoIDt, "Some Numerical Solutions of the Boundary Layer Equations for SF6 Arc”, Proc. IEEE, Vol 59, pp 493-501, 1971.

2. W. Hermann AND K. Ragaller, "Theoretical Description of the Current Interruption in High Voltage Gas Blast Circuit Breakers", IEEE Tran. PAS, Vol PAS-96, pp 1546-1555, 1977.

3. J. J. Lowke AND H. E. LEe, "A Numerical Study of a Two Dimensional Circuit Breaker Arc during Current Interruption", Proc. of gas discharge and their applications, Oxford, pp 54-56, 1985.

4. S. K. Chan, M. T. C. FAng And M. D. Cowley, "The DC Arc in a Supersonic Flow", IEEE Tran., Plasma Science, Vol PS-6, pp 394-405, 1978.

5. H.J. Choe, M.-Y. Ahn, K.D. Song, K.-Y. Park, S.-K. Park, Flow field computation for simplified high voltage gas circuit breaker model by upwind meshfree method, Jpn. J. Appl. Phys. 45 (2006), 9247-9253.

6. S.-K. Park, K.-Y. Park, H.J. Choe, Flow field computation for the high voltage gas blast circuit breaker with the moving boundary, Comput. Phys. Commun., to appear 